\title{
BMJ Open Incidence and characteristics of ventilator-associated pneumonia in a regional non-tertiary Australian intensive care unit: protocol for a retrospective clinical audit study
}

\author{
Kirsty Watson, ${ }^{1,2}$ Luke J Heales, ${ }^{3,4}$ Jeremy Fernando, ${ }^{1,5}$ Josephine Reoch, \\ Elise Tan, ${ }^{1}$ Karen Smith, ${ }^{1}$ David Austin, ${ }^{1}$ Anestis Divanoglou ${ }^{3,6}$
}

To cite: Watson K, Heales LJ, Fernando J, et al. Incidence and characteristics of ventilatorassociated pneumonia in a regional non-tertiary Australian intensive care unit: protocol for a retrospective clinical audit study. BMJ Open 2018;8:e021733. doi:10.1136/ bmjopen-2018-021733

- Prepublication history for this paper is available online. To view these files, please visit the journal online (http://dx.doi. org/10.1136/bmjopen-2018021733).

Received 18 January 2018

Revised 2 July 2018

Accepted 17 July 2018
D) Check for updates

(C) Author(s) (or their employer(s)) 2018. Re-use permitted under CC BY-NC. No commercial re-use. See rights and permissions. Published by BMJ.

For numbered affiliations see end of article.

\section{Correspondence to} Dr Jeremy Fernando; Jeremy.Fernando@health.qld. gov.au

\section{ABSTRACT}

Introduction Ventilator-associated pneumonia (VAP) is a medical complication associated with prolonged mechanical ventilation. Most studies looking at VAP originate from major, tertiary intensive care units (ICUs). Our understanding of VAP in regional hospitals is limited. Given that patient characteristics often differ between metropolitan and regional centres, it is important to investigate VAP in a regional non-tertiary ICU. This project will establish and report the incidence, case characteristics and outcomes including mortality and length of stay related to VAP in a regional non-tertiary Australian ICU. Furthermore, it will compare the incidence of VAP in accordance with consultant diagnosed cases in the medical record, and by a post hoc screening of all cases against a list of previously published diagnostic criteria. Methods and analysis This retrospective clinical audit study will screen medical records from the period 1 January 2013 to 31 December 2016. All cases requiring mechanical ventilation for $\geq 72$ hours will be screened against previously reported diagnostic criteria for VAP. At the same time, their medical records will be screened for a documented diagnosis of VAP.

Ethics and dissemination This study has been granted ethical approval from the Central Queensland Hospital and Health Service (CQHHS) Human Research Ethics Committee (HREC/17/QCQ/11) and the Central Queensland University Human Research Ethics Committee (H17/05102). This study will be submitted for publication in a peer-reviewed scientific journal and presented at internal workshops (within Queensland Health) and national and/or international scientific conferences.

\section{INTRODUCTION}

Critically unwell patients often require intubation and ventilation for prolonged periods of time. Ventilator-associated pneumonia (VAP) is a recognised complication of mechanical ventilation with multiple predisposing factors. These include secretion retention, inadequate cough, immunosuppression, respiratory tract infection, poor oral

\section{Strengths and limitations of this study}

- Study findings will provide an improved understanding of the magnitude and impact of ventilator-associated pneumonia (VAP) in a regional non-tertiary intensive care unit (ICU) setting. This is of particular importance given that regional settings are different to metropolitan in terms of sociodemographics, comorbidities and access to healthcare.

- The study aims to establish and report the incidence, case characteristics and outcomes related to VAP in a regional non-tertiary Australian ICU.

- The study comprises a retrospective clinical audit of electronic patient records over a 4-year period.

- It will involve a systematic evaluation of VAP in accordance with detailed and previously published criteria.

- Inclusion of both diagnostic criteria-based diagnosis and medical consultant diagnosis will provide insight into potential under-reporting or over-reporting of VAP in clinical practice in a regional setting.

hygiene and poor patient positioning. VAP increases morbidity and intensive care unit (ICU) mortality, ${ }^{12}$ as well as hospital length of stay (LOS), which subsequently increases healthcare cost. ${ }^{34}$ For example, Kollef et $a l^{4}$ reported mean hospital costs of US\$99598 for patients with VAP compared with US\$59770 for patients without VAP.

There is currently no consensus on the diagnosis and definition of VAP. ${ }^{5}$ This is due to the lack of clinically relevant diagnostic criteria. A recent study by Elliott et at ${ }^{6}$ reported a list of diagnostic criteria to establish the VAP incidence in a cohort of 10 Australian ICUs. These criteria evolved through a modified Delphi study and were reviewed by the Quality and Safety Committee of the Australian and New Zealand Intensive Care Society. ${ }^{6}$ 
To date, the incidence of VAP, its associated characteristics, mortality and morbidity has not been established in a regional non-tertiary ICU setting within Australia or New Zealand. Patients in regional settings within Australia are known to have higher rates of comorbidities than those in metropolitan centres. ${ }^{7}$ It is well documented that the presence of serious comorbidities are known risk factors for $\mathrm{VAP}^{8}$ and are generally associated with case fatality, ${ }^{9}$ hence the importance of this study.

The study objectives are: (1) to identify the incidence of VAP in a regional ICU over a 4-year period (primary aim); (2) to compare a checklist-based definition of VAP to an ICU consultant diagnosis of VAP; (3) to explore the patient characteristics of VAP-associated morbidity; (4) to establish mortality rates of cases with VAP and (5) to compare LOS and discharge destination between patients with VAP and patients without VAP in regional Australia.

A better understanding of the incidence of VAP, mortality rate and case characteristics will provide an improved insight into the magnitude of VAP in our regional setting and allow the development of targeted and effective management strategies. Finally, the completion of this study will allow comparison of our findings with metropolitan populations, which to our knowledge has not been previously reported.

\section{METHODS AND ANALYSIS}

\section{Study design}

Retrospective clinical audit of electronic patient records.

\section{Inclusion and exclusion criteria}

Inclusion criteria are all cases $\geq 16$ years of age, admitted to the ICU, Rockhampton Hospital, Central Queensland, who received invasive mechanical ventilation (MV) for $\geq 72$ hours during the period 1 January 2013 to 31 December 2016. Cases will be excluded if they were diagnosed with a community or hospital-acquired pneumonia prior to or during the first 72 hours of MV.

\section{Case identification}

The local database administrator will use an automated computer algorithm to identify all cases in accordance with our inclusion criteria (described above). Following case identification, the local database administrator will work in collaboration with the central database administrator to extract all relevant data of the identified cases. These data will form the master database.

\section{Checklist-based definition of VAP}

In order to achieve the primary aim of this study, all identified cases will be screened based on a previously published checklist of diagnostic criteria. ${ }^{6}$ Two investigators with extensive clinical experience in the diagnosis and management of VAP will independently evaluate each case and will classify them as VAP or non-VAP. Any discrepancies between the two investigators will be resolved by discussion with an independent third investigator-an
ICU consultant with extensive clinical experience. Cases will be diagnosed with VAP based on a checklist by fulfilling four objective, previously published diagnostic criteria $^{6}$ in a 48 -hour window. These four criteria will be applied in the following priority order:

1. $\mathrm{PaO}_{2} / \mathrm{FiO}_{2}$ ratio $\leq 300 \mathrm{~mm} \mathrm{Hg}$ : deterioration in gas exchange over past 24 hours in the absence of cardiac or pulmonary disease.

2. Inflammatory response $\geq$ one of the following (in the absence of immunocompromise):

a. $\uparrow$ Temperature: new and persistent (past 24 hours) elevated body temperature $38^{\circ} \mathrm{C}$ (or $>37.5^{\circ} \mathrm{C}$ if concurrent antipyretic medication administration);

b. White cell count (WCC): $\leq 4$ or $\geq 12$ cells $10^{9} / \mathrm{L}$ for two consecutive days;

c. $\uparrow$ Inflammation: elevated serum inflammatory markers: C reactive protein $(>100 \mathrm{mg} / \mathrm{L})$ or procalcitonin $(>2.5 \mathrm{ng} / \mathrm{L})$.

3. Sputum changes: a change in sputum characteristics, either increased volume or colour change (yellow or green).

4. Chest C-ray (CXR) infiltrates: new localised or diffuse infiltrates on a single CXR (not explained by cardiac or pulmonary disease).

Originally, Elliott $e t a t$ included five diagnostic criteria. However, the fifth criterion 'Microbial growth in tracheal secretions obtained by tracheal suctioning or bronchoscopy (ie, $>25$ neutrophils per low power field or equivalent)' did not contribute to their explanatory model. ${ }^{6}$ Based on that, we decided to exclude it from the list of diagnostic criteria of our study.

\section{Consultant-based diagnosis of VAP}

To achieve the second aim, we will compare the findings from our checklist-based identification of VAP (defined above) to a consultant-based diagnosis of VAP identified from the medical records of all cases. To accomplish this, two additional investigators who are not medical consultants (to remove potential bias) will independently conduct an electronic search of the medical records of all cases to identify where a medical consultant has made a clinical diagnosis of VAP. To remove potential bias and standardise the search, keywords including 'ventilator-associated pneumonia', OR 'VAP', OR 'pneumonia' will be used. The inclusion of the word 'pneumonia' is intended to aid the identification of cases where the condition is clearly described in the medical records but the exact phrase 'ventilator-associated pneumonia' or abbreviation 'VAP' is missing. This process requires minimal individual assessment and judgement. Discrepancies between the two investigators will be resolved through discussion until consensus is reached. The process for case identification is presented in the flow chart (figure 1).

\section{Methods for aims $\mathbf{3} \mathbf{- 5}$}

To explore the patient characteristics of VAP-associated morbidity (aim 3), our model will include demographic and clinical characteristics, including microbiology 


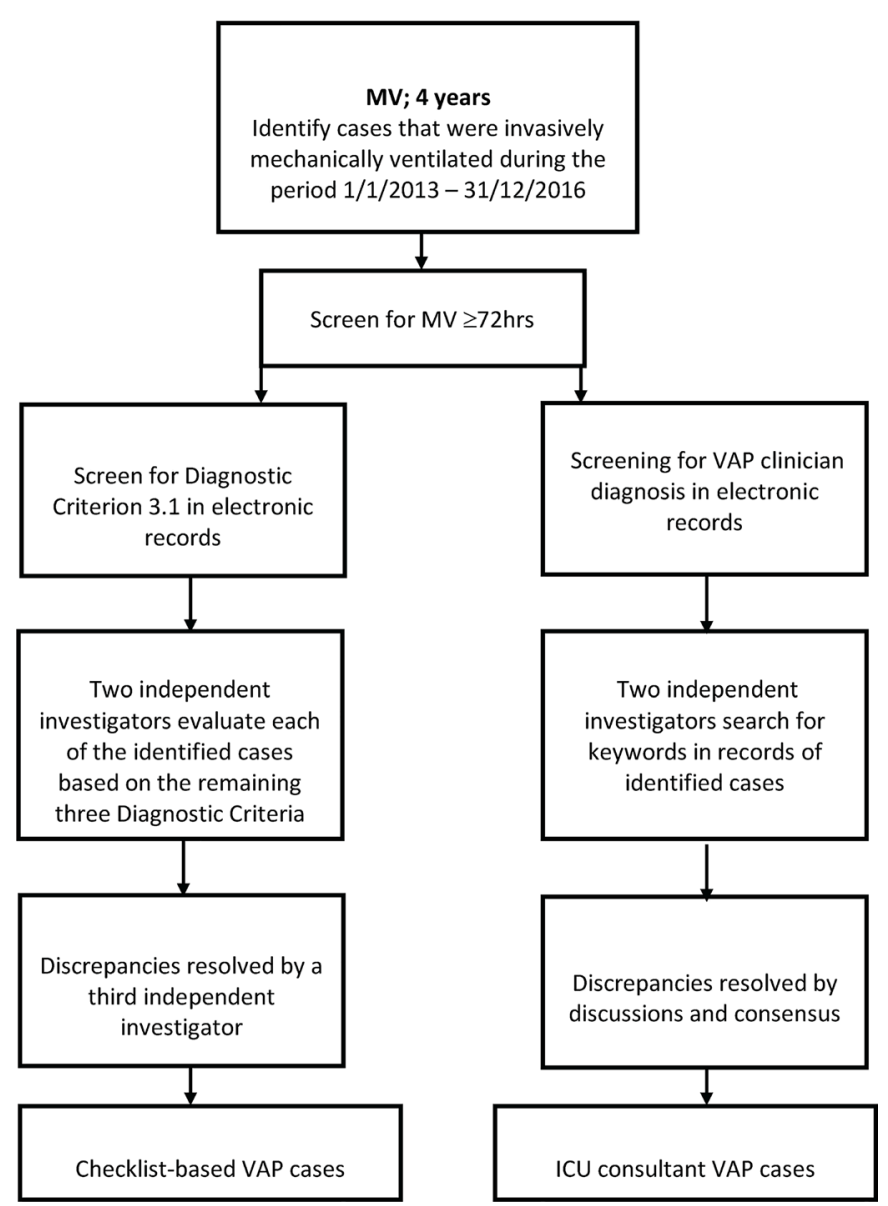

Figure 1 Flow chart presenting the case identification and screening processes. ICU, intensive care unit; $\mathrm{MV}$, mechanical ventilation; VAP, ventilator- associated pneumonia.

related to VAP; Acute Physiology and Chronic Health Evaluation II (APACHE II) Score based on the worst values during the initial 24 hours in ICU, and Charlson Comorbidities Index on admission to ICU. These data will be extracted from the electronic medical record.

We will calculate and report crude and adjusted mortality rates for both VAP and non-VAP cases for day 30 and 60 in ICU, as well as for the total ICU stay (aim 4).

In order to investigate aim 5, we will extract and compare data about LOS in ICU, total LOS at the Rockhampton Hospital and discharge destination, for both VAP and non-VAP cases who meet our inclusion criteria.

\section{Sample size}

Due to the clinical audit design of this study, it is not possible to calculate our sample size a priori. Based on an estimate, there are approximately 80 cases per year requiring $\geq 72$ hours of invasive MV. We estimated our sample size to be approximately 320 cases over the 4-year period. Our estimated sample size is consistent with previous published literature regarding VAP within a tertiary facility. ${ }^{6}$

\section{Data analysis plan}

Descriptive data will be presented as: $\mathrm{n}(\%)$, mean and SD or median and IQR. Statistical significance will be set at alpha $<0.05$. Differences in proportions will be examined by $\mathrm{Chi}^{2}$ test or Fisher's exact test depending on the number of cases for each outcome measure. Independent student's t-test will be used to investigate betweengroup differences. For ordinal variables or in cases of non-normal distribution, the Mann-Whitney $\mathrm{U}$ test will be used.

Similar to the study by Elliott, et $a l,{ }^{6}$ we will report VAP incidence based on the cases who meet all four diagnostic criteria, and on cases with a consultant VAP diagnosis in their electronic records. The VAP incidence rate will be calculated as the number of cases with VAP/total number of ventilator days for identified cases who meet the inclusion criteria $\mathrm{x} 1000$. Also, the VAP incidence rate will be calculated and reported as the number of cases with VAP/ total number of cases who meet the inclusion criteria x1000. Adjusted mortality rates for VAP and non-VAP cases will be computed through Poisson risk model with robust variance estimate adjusted for gender, APACHE II score and Charlson Comorbidities Index on admission.

The reliability of both case identification processes (ie, criteria-based diagnosis and record screening for consultant diagnosis of VAP) will be evaluated using Kappa statistics for categorical data. Interinvestigator reliability will be established between the two independent investigators when applying the predetermined diagnostic criteria (VAP or non-VAP) and the two independent investigators when searching for medical consultant diagnosis (VAP or non-VAP). Case identification reliability will be considered as poor $(<0.00)$, slight $(0.00-0.2)$, fair (0.21-0.4), moderate $(0.41-0.6)$, substantial (0.61-0.8) or almost perfect $(0.81-1.0) .^{10}$

In particularly for aim 3, binary logistic regression analysis will be used to explore which characteristics increase the probability for VAP onset. Analysis will be conducted in a stepwise approach and will include age, gender, BMI, APACHE II score and Charlson Comorbidities Index on admission to ICU, and results will be presented as ORs with $95 \%$ CIs.

Results will be presented for VAP and non-VAP subgroups resulting from checklist-based and consultant-based procedures. All statistical analyses will be performed with the SPSS software (Statistical Package for the Social Sciences, V.24.0, Chicago, Illinois, USA).

\section{Patient and public involvement}

Patients or the public were not involved in the design of this study.

\section{ETHICS AND DISSEMINATION}

This study has been granted ethical approval from the Central Queensland Hospital and Health Service (CQHHS) Human Research Ethics Committee (HREC/17/QCQ/11) and the Central Queensland 
University Human Research Ethics Committee (H17/05102). We expect to complete data extraction in September 2018 and aim to complete the study 12 months later. We intend to publish in a peer-reviewed scientific journal. Our research outcomes will also be presented to the clinicians of the Rockhampton Hospital ICU, to help inform best practice. We will also share our research outcomes with the Queensland Intensive Care Network. Lastly, we will present the outcomes of this research in the form of an oral or poster presentation at a national and/or international scientific conference, such as the Australian and New Zealand Intensive Care/Australian College of Critical Nurses Annual Scientific Meeting.

\section{SIGNIFICANCE}

Our project will establish and report the incidence, case characteristics and outcomes related to VAP in a regional non-tertiary Australian ICU. This will provide an improved understanding of the magnitude of this common and expensive complication. It is possible that the different sociodemographic and health profile of the population in regional Queensland (as compared with metropolitan centres), may have a strong impact on VAP incidence and outcomes including mortality.

The inclusion of both diagnostic criteria-based diagnosis and medical consultant diagnosis will be used to identify potential under-reporting or over-reporting of VAP in clinical practice. A better understanding of the incidence of VAP and characteristics associated with VAP in a regional non-tertiary ICU provides the potential to systematically monitor VAP cases, allowing early detection and treatment.

\section{Author affiliations}

${ }^{1}$ Intensive Care Unit, Rockhampton Hospital, Rockhampton, Queensland, Australia ${ }^{2}$ Physiotherapy, Rockhampton Hospital, Rockhampton, Queensland, Australia

${ }^{3}$ School of Health, Medical and Applied Sciences, Central Queensland University, Rockhampton, Queensland, Australia

${ }^{4}$ School of Biomedical Sciences, University of Queensland, Brisbane, Queensland, Australia

${ }^{5}$ Rural Clinical School, University of Queensland, Rockhampton, Queensland, Australia

${ }^{6}$ School of Health Sciences, University of Iceland, Reykjavik, Iceland

Acknowledgements The authors would like to thank Rosalind and Doug Elliot for providing advice and sharing resources.
Contributors This study was conceived by KW, JR and ET. AD and LJH provided methodological support. KW, JF, JR, ET, KS and DA provided their clinical expertise. $A D$ is the chief researcher and together with KW and JR, they are the principle investigators of the study. All authors participated in collaborative meetings about the study design. JR designed the data extraction process. KW, AD and LJH drafted the ethics application with input from JR, JF and DA. AD, LJH and JF drafted the paper and KW, JR, ET, KS and DA provided feedback to the manuscript. All authors approved the final version of the manuscript.

Funding The authors have not declared a specific grant for this research from any funding agency in the public, commercial or not-for-profit sectors.

Competing interests None declared.

Patient consent Not required.

Ethics approval This study has been granted ethical approval from the Central Queensland Hospital and Health Service (CQHHS) Human Research Ethics Committee (HREC/17/QCQ/11) and the Central Queensland University Human Research Ethics Committee (H17/05-102).

Provenance and peer review Not commissioned; externally peer reviewed.

\section{Data sharing statement None.}

Open access This is an open access article distributed in accordance with the Creative Commons Attribution Non Commercial (CC BY-NC 4.0) license, which permits others to distribute, remix, adapt, build upon this work non-commercially, and license their derivative works on different terms, provided the original work is properly cited, appropriate credit is given, any changes made indicated, and the use is non-commercial. See: http://creativecommons.org/licenses/by-nc/4.0/.

\section{REFERENCES}

1. Melsen WG, Rovers MM, Koeman M, et al. Estimating the attributable mortality of ventilator-associated pneumonia from randomized prevention studies. Crit Care Med 2011;39:2736-42.

2. Bekaert M, Timsit JF, Vansteelandt S, et al. Attributable mortality of ventilator-associated pneumonia: a reappraisal using causal analysis. Am J Respir Crit Care Med 2011;184:1133-9.

3. Safdar N, Dezfulian C, Collard HR, et al. Clinical and economic consequences of ventilator-associated pneumonia: a systematic review. Crit Care Med 2005;33:2184-93.

4. Kollef $\mathrm{MH}$, Hamilton $\mathrm{CW}$, Ernst FR. Economic impact of ventilatorassociated pneumonia in a large matched cohort. Infect Control Hosp Epidemiol 2012;33:250-6.

5. Rello J, Lisboa T, Koulenti D. Respiratory infections in patients undergoing mechanical ventilation. Lancet Respir Med 2014;2:764-74.

6. Elliott D, Elliott R, Burrell A, et al. Incidence of ventilator-associated pneumonia in Australasian intensive care units: use of a consensusdeveloped clinical surveillance checklist in a multisite prospective audit. BMJ Open 2015;5:e008924-e24.

7. Australian Institute of Health and Welfare AG. 2017 http://www.aihw. gov.au/.

8. Alp E, Voss A. Ventilator associated pneumonia and infection control. Ann Clin Microbiol Antimicrob 2006;5:7.

9. Tsioutis C, Kritsotakis El, Karageorgos SA, et al. Clinical epidemiology, treatment and prognostic factors of extensively drugresistant Acinetobacter baumannii ventilator-associated pneumonia in critically ill patients. Int J Antimicrob Agents 2016;48:492-7.

10. Landis JR, Koch GG. The measurement of observer agreement for categorical data. Biometrics 1977;33:159-74. 